\title{
Radiolabeled Antibodies, Albumin and Antimony Sulfide Colloid: A Comparison as Lymphoscintigraphic Agents
}

\author{
RICHARD L. WAHL, ${ }^{*}$ MONICA LIEBERT, BARRY S. WILSON \\ and NEIL A. PETRY \\ University of Michigan Medical Center, Department of Internal Medicine, Division of Nuclear Medicine, \\ Ann Arbor, MI 48109-0028, U.S.A.
}

(Received 28 July 1987)

\begin{abstract}
The kinetics of lymph node and systemic uptake of members of three different classes of lymphoscintigraphic agents were studied in normal laboratory rats. ${ }^{99 \mathrm{~m}} \mathrm{Tc}$ antimony trisulfide colloid (TcSbC), ${ }^{99 \mathrm{~m}} \mathrm{Tc}$ human serum albumin (TcHSA), ${ }^{125} \mathrm{I}$ 5G6.4 (a murine IgG2ak monoclonal antibody), ${ }^{125} \mathrm{I} 763.24 \mathrm{~T}$ (a murine $\operatorname{IgG}_{1}$ ), and ${ }^{125} \mathrm{I}$ FT166 (a murine IgM monoclonal) all current or potential lymphoscintigraphic agents, were injected subcutaneously into the hind foot pads of healthy rats. Ipsilateral and contralateral popliteal lymph nodes were sampled up to $4 \mathrm{~h}$ post-injection. Subcutaneous injection resulted in far higher nodal uptake for all agents than i.v. delivery with ipsilateral popliteal node/blood ratios $1 \mathrm{~h}$ postsubcutaneous injection of: for TcSbC (1900) $>{ }^{125}$ I IgM (497) $>$ TcHSA (72) $>{ }^{125}$ I Intact IgG2a or ${ }^{125}$ I $\mathrm{IgG}_{1}$ at approximately 10 . Thus, while all agents achieve popliteal node/blood ratios far greater than unity, TcSbc has the greatest absolute and relative nodal accumulation, greater than the ${ }^{125}$ IgM monoclonal antibody and TcHSA. Uptake of the intact ${ }^{125} \mathrm{I} \mathrm{IgG}$ antibodies is lowest. These data suggest that TcSbC in particular, as well as TcHSA and IgM may be most useful as non-specific nodel imaging agents, while the lower background activity of the IgGs may make targeting specific antigen in nodes more feasible.
\end{abstract}

\section{Introduction}

Lymphoscintigraphy, the intradermal or subcutaneous injection of a radiocolloid, has become an increasingly common technique in the past several years for mapping potential sites of nodal metastases and for predicting involvement with tumor (Croll et al., 1983). A considerable number of agents have been cvaluated as possible lymphoscintigraphic agents (Bergvist et al., 1983). ${ }^{99 \mathrm{~m}} \mathrm{Tc}$ antimony trisulfide colloid ((TcSbC) has been used extensively clinically, while we have more recently been using ${ }^{99 \mathrm{~m}} \mathrm{Tc}$ human serum albumin (TcHSA) for scanning (Croll et al., 1983; Froelich et al., 1984; Eberbach et al., 1987). Radiolabeled antibodies, and most recently monoclonal antibodies have also been suggested as potentially useful imaging agents and have shown promising results in animal models and in some clinical trials (DeLand et al., 1980; Weinstein et al., 1983; Carrasquillo et al., 1986; Wahl et al., 1987).

*All correspondence should be addressed to: Richard L. Wahl, M.D., University of Michigan Medical Center, Division of Nuclear Medicine, 1500 East Medical Center Drive, Ann Arbor, MI 48109-0028, U.S.A.
While some information is available regarding the rate of egress of monoclonal antibodies injected subcutaneously, little is known regarding the kinetics of uptake of these agents into normal lymph nodes or if the larger IgM is handled differently (Bergqvist et al., 1983; Wahl et al., 1985). Similarly, relatively little is known regarding comparisons of noncolloidal TcHSA, which has been clinically useful in our practice, with the TcSbC product available for investigational use in the U.S. (Bergqvist et al., 1983; Eberbach et al., 1987). In fact, we have recently demonstrated that small molecules such as ${ }^{99 \mathrm{~m}} \mathrm{Tc}$ MDP used for bone scanning can reach relatively high levels in regional lymph nodes (following s.c. administration) suggesting that the colloidal state may not be a necessary prerequisite to achieving significant levels of uptake of radiopharmaceuticals to regional lymph nodes as has been previously suggested (Croll et al., 1983; Wallis et al., 1987). This study was designed to delineate the rate and extent of lymph node and systemic uptake of these current and potential lymphoscintigraphic agents.

\section{Methods}

The lymphoscintigraphic agents studied included: 


\section{$T c S b C$}

This agent, supplied in kit form by Cadema, was prepared by adding $10-15 \mathrm{mCi}$ of $\left[{ }^{99 \mathrm{~m}} \mathrm{Tc}\right]$ pertechnetate to a kit supplied by Cadema. Fifty to $100 \mu \mathrm{Ci}$ was given per animal. Purity of over $90 \%$ was typical by acetone silica gel thin layer chromatography (Gelman, Ann Arbor, Mich.).

\section{TcHSA}

This was made by adding $4 \mathrm{mCi}$ of ${ }^{99 \mathrm{~m}} \mathrm{Tc}$ to $7 \mathrm{mg}$ of human serum albumin. This was provided in kit form by Medi-Physics. This reagent was $98 \%$ devoid of free $\mathrm{TcO}_{4}^{-}$by acetone silica gel thin layer chromatography (Gelman, Ann Arbor, Mich.). One hundred $\mu \mathrm{Ci}$ was given per animal (approximately $70 \mu \mathrm{g}$ ).

\section{$5 G 6.4$}

5 G6.4 is a murine IgG2ak reactive with ovarian and other epithelial carcinomas (Wahl et al., 1986). This agent was labeled using the iodogen method in which $100 \mu \mathrm{g}$ was reacted with $1 \mathrm{mCi}$ of ${ }^{125} \mathrm{I}$ (ICN). Yields of over $70 \%$ were typical, with $>95 \%$ purity following Biogel (Biorad) P-60 chromatography. Nine to ten $\mu \mathrm{Ci}$ were given per animal.

Similar iodination conditions were used for FT 166, a murine IgM reactive with bladder carcinomas (4-5 $\mu \mathrm{Ci}$ injected per animal) (Liebert), and 763.24T (10-11 $\mu \mathrm{Ci}$ injected per animal), a murine IgG1k (Wilson et al., 1982). The purity of these antibodies was confirmed by SDS polyacrylamide gel electrophoresis (Laemmli, 1970).

Adult, female Sprague Dawley rats were selected for study. All lymphoscintigraphic agent injections were made either intravenously by the femoral vein or subcutaneously into one hind foot pad. Animals were studied in groups of 3-7. Injection volumes were $100 \mu \mathrm{L}$ or less. Tissues, including popliteal lymph nodes (ipsilateral and contralateral), ipsilateral and contralateral inguinal lymph nodes, blood, liver and lower leg muscle were obtained at selected intervals post-injection, weighted and counted using standard tissue processing techniques (Wahl et al., 1984). Radioactive decay was corrected for and the results were expressed as \% kg-injected dose/g. Lymph node/blood ratios were determined by dividing lymph node uptake $(\% \mathrm{~kg}$ dose $/ \mathrm{g})$ by blood uptake $(\% \mathrm{~kg}$ dose/g). 763.24T and FT166 were studied following s.c. administration only.

Statistical analysis was by the Student's $t$ test and analysis of variance methods.

\section{Results}

\section{${ }^{99 m}$ Tc antimony trisulfide colloid}

From 30 min until $4 \mathrm{~h}$ after the injection of TcSbC, there was a dramatic increase in the uptake of the agent in the ipsilateral popliteal nodes. By $4 \mathrm{~h}$, the percent $\mathrm{kg}$-dose/g was $231.6 \pm 40.2$ (SD) (Fig. 1). When given intravenously, the same dose of TcSbC produced nodal uptake of $0.0551 \pm 0.0106$ (Table 1).
LYMPHOSCINTIGRAPHIC AGENTS

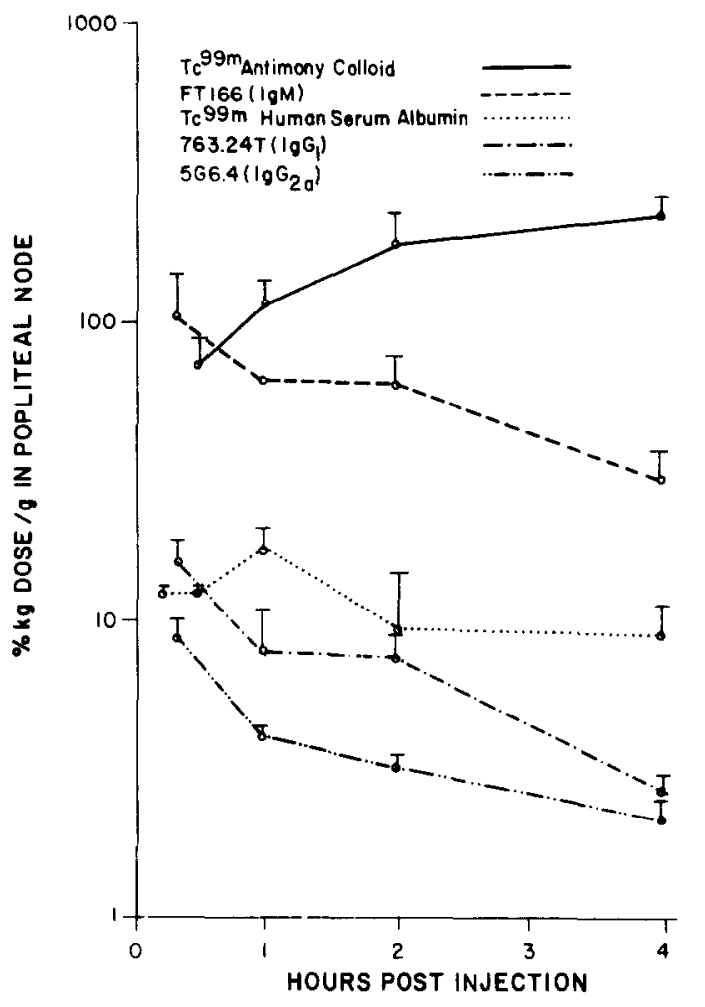

Fig. 1. Semi-log plot of the uptake of five possible lymphoscintigraphic agents over time to the popliteal lymph nodes. Injections were all made subcutaneously to the hind foot pad.

Following i.v. administration, there is high accumulation of TcSbC in the liver and spleen, compatible with the typical behavior of colloid (Table 1). Ipsilateral popliteal node/blood ratios reached $4070 / 1$ by $4 \mathrm{~h}$ after s.c. injection.

\section{${ }^{99 m}{ }^{9 c} H S A$}

At $10 \mathrm{~min}$ following the injection of TcHSA, popliteal node uptake was $12.359 \pm 0.61$, with a node/blood ratio of 220.7. The absolute uptake in the right popliteal node increased to peak at $1 \mathrm{~h}(18.24$ \pm 7.53 ), and then fell to $9.33 \pm 2.16$ by $4 \mathrm{~h}$ postinjection. The popliteal node/blood ratio also fell to $38.3 / 1$ by $4 \mathrm{~h}$ post-injection (Fig. 1 and Table 2). Blood levels were considerably higher than for TcSbC following s.c. administration. Note that liver uptake following i.v. administration is considerably less than for antimony colloid. When TcHSA was given by the i.v. route, in contrast to the s.c. route, only minimal uptake was seen in popliteal lymph nodes (Table 2).

\section{${ }^{125}$ I 5 G6.4 (intact IgG2ak)}

This agent behaved in a similar fashion to TcHSA, in that nodal uptake ipsilateral to the injected foot peaked early and then declined. Maximal node/blood ratios were achieved soon after injection as well. These node/blood ratios fell from $74 / 1$ at $20 \mathrm{~min}$ to 

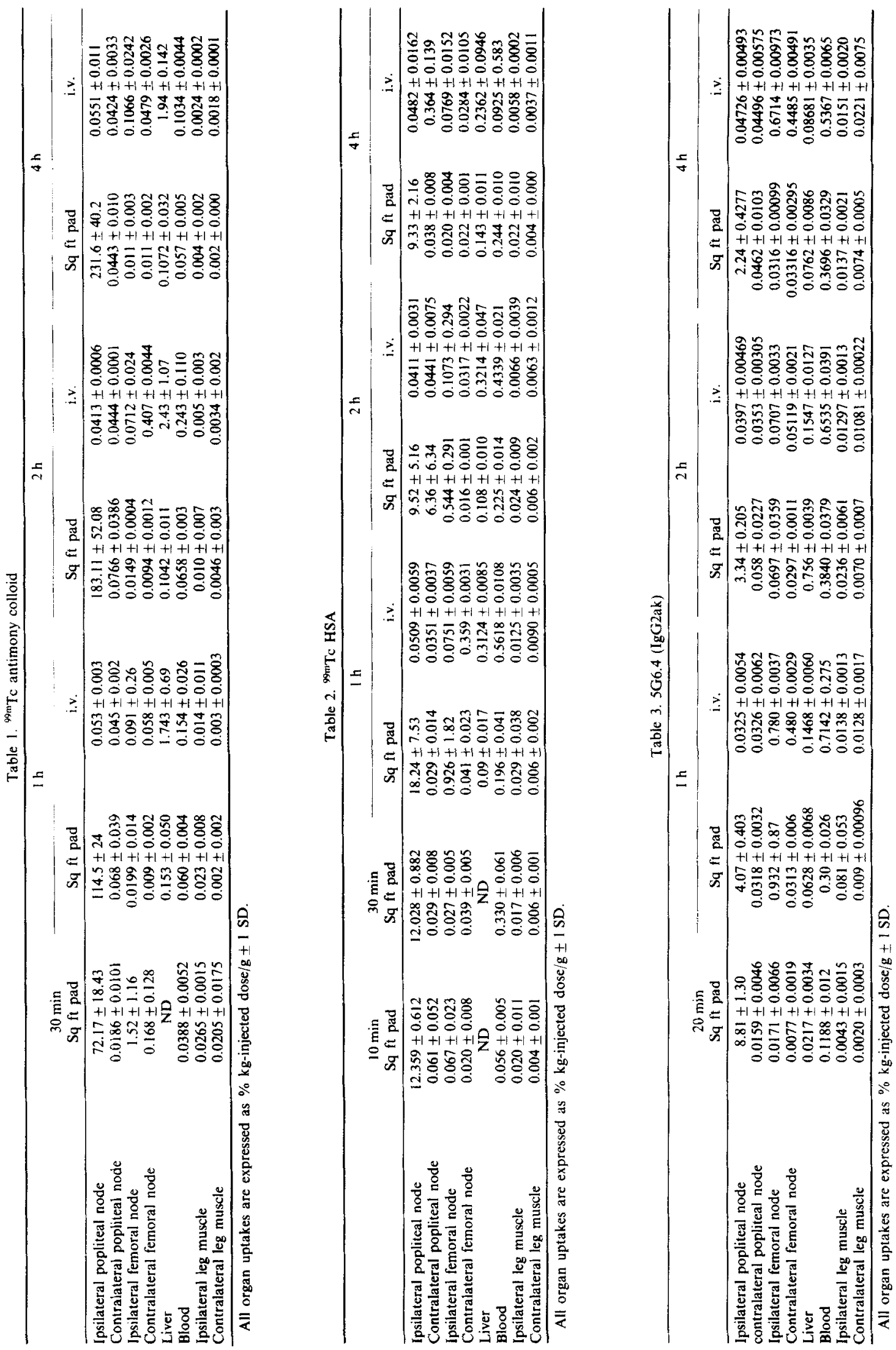
Table 4. ${ }^{125} \mathrm{I} 763.24 \mathrm{~T}\left(\mathrm{IgG}_{1}\right)$

\begin{tabular}{lcccc}
\hline & $20 \mathrm{~min}$ & $1 \mathrm{~h}$ & $2 \mathrm{~h}$ & $4 \mathrm{~h}$ \\
\hline Ipsilateral popliteal node & $15.82 \pm 2.73$ & $7.86 \pm 2.99$ & $7.60 \pm 1.58$ & $2.82 \pm 0.40$ \\
Contralateral popliteal node & $0.058 \pm 0.027$ & $0.057 \pm 0.020$ & $0.085 \pm 0.026$ & $0.052 \pm 0.008$ \\
Ipsilateral femoral node & $1.34 \pm 1.32$ & $0.426 \pm 0.247$ & $0.239 \pm 0.180$ & $0.584 \pm 0.328$ \\
Contralateral femoral node & $0.033 \pm 0.006$ & $0.031 \pm 0.008$ & $0.036 \pm 0.001$ & $0.048 \pm 0.003$ \\
Liver & $0.03 \pm 0.007$ & $0.089 \pm 0.004$ & $0.130 \pm 0.012$ & $0.152 \pm 0.015$ \\
Blood & $0.190 \pm 0.042$ & $0.484 \pm 0.044$ & $0.682 \pm 0.088$ & $0.761 \pm 0.190$ \\
Ipsilateral leg muscle & $0.005 \pm 0.000$ & $0.006 \pm 0.001$ & $0.009 \pm 0.002$ & $0.011 \pm 0.002$ \\
Contralateral leg muscle & $0.033 \pm 0.006$ & $0.007 \pm 0.001$ & $0.008 \pm 0.000$ & $0.011 \pm 0.002$ \\
\hline
\end{tabular}

All organ uptakes are expressed as $\% \mathrm{~kg}$-injected dose $/ \mathrm{g} \pm \mathrm{I} \mathrm{SD}$ (following s.c. administration to a hind foot pad)

6.1/1 at $4 \mathrm{~h}$ post-injection (Table 3). Absolute popliteal node activity also dropped with time. At $20 \mathrm{~min}$ post ${ }^{125}$ I 5G6.4 injection the ipsilateral popliteal node activity was $8.81 \pm 1.30$, but fell to $2.24 \pm 0.43$ at $4 \mathrm{~h}$ after injection.

\section{${ }^{125} I 763.24 T$ (IgG $1 k$ )}

This 150,000 dalton mouse monoclonal behaved very similarly to the ${ }^{125} \mathrm{I} 5 \mathrm{G} 6.4$ antibody. At $20 \mathrm{~min}$ post-s.c. injection $15.82 \pm 2.73 \% \quad \mathrm{~kg}$-dose $/ \mathrm{g}$ was located within the ipsilateral poplitcal lymph node, while by $4 \mathrm{~h}$ post-injection, this had dropped to 2.82 \pm 0.40 . Ipsilateral popliteal node/blood ratios fell from $83.4 / 1$ to $3.71 / 1$ at $4 \mathrm{~h}$ post-injection (Table 4 ).

\section{${ }^{125}$ I FT166 (IgM)}

This 900,000 dalton mouse monoclonal behaved differently from the ${ }^{125} \mathrm{I}$ IgG's in terms of nodal uptake. For example, at $20 \mathrm{~min}$ after s.c. injection, the ipsilateral popliteal node harbored $102.1 \pm 40.3 \%$ $\mathrm{kg}$-dose $/ \mathrm{g}$ and at $4 \mathrm{~h}$ still retained $32.4 \pm 6.4 \% \mathrm{~kg}-$ dose/g (Fig. 1). Ipsilateral node/blood ratios also were much higher than with the ${ }^{125} \mathrm{I} \mathrm{IgG}$ antibodies, with node/blood ratios of $1173 / 1$ at $20 \mathrm{~min}$, and $224.7 / 1$ at $4 \mathrm{~h}$ post-injection (Table 5).

\section{Discussion}

Our study compares several diverse colloidal and non-colloidal lymphoscintigraphic agents in the same animal system. These data shows that $\mathrm{TcSbC}$ is taken up to a much higher level in lymph nodes than are ${ }^{125} \mathrm{I}$ IgG antibodies or TcHSA. In fact, node/blood ratios in excess of several thousand are seen with TcSbC. The ${ }^{125}$ I IgM, FT166, is taken up in the popliteal nodes far more than TcHSA or either the ${ }^{125} \mathrm{IgG}_{1}$ or the ${ }^{125} \mathrm{I} I g G 2 a$ intact antibodies. At early time points, the ${ }^{125}$ IgM has slightly higher nodal uptake than the $\mathrm{TcSbC}$. The TcHSA has a behavior intermediate between the ${ }^{125}$ I IgM and the ${ }^{125}$ I IgG's. This was somewhat surprising, in that monomeric allumin would have a molecular weight in the 70,000 range, smaller than an intact antibody and might be expected to be less highly retained than the larger IgG's. Radiochromatographic TSK 3000 HPLC tracings of the TcHSA product we use routinely show an elution volume slightly greater than that of $\mathrm{IgG}$, in the range expected for monomeric albumin. This indicates that the TcHSA is not aggregated into a larger moiety.

The ${ }^{125} \mathrm{I}$ IgG antibodies, an IgG2a 5G6.4 (reactive with ovarian cancer) (Wahl et al., 1986) and the $\mathrm{IgG}_{1}$ 763.24T (reactive with melanoma) (Wilson et al., 1982) both have kappa light chains, and both behave similarly in vivo. While non-specific uptake of antibodies has been described in human imaging studies, the non-specific retention of these IgG agents appears relatively low, as at $4 \mathrm{~h}$ the popliteal nodes had less than 1/100th the uptake that antimony colloid possessed (Carrasquillo et al., 1986; Engelstad et al., 1986). TcHSA was retained to a higher degree than the ${ }^{125} \mathrm{I}$ IgG antibody in the nodes. The mechanism for these differences is unclear at present. Certainly, some antibody binding to lymph nodes could be due to $\mathrm{F}-\mathrm{C}$ receptor binding to mononuclear cells in the node, however the reason for higher TcHSA uptake is unclear (Hopf et al., 1976).

Presumedly, much of the activity we see in lymph nodes over time is related to transient passage of the ${ }^{125} \mathrm{I} \mathrm{IgG}$ antibodies through the nodes. The TcSbC appears to be actively sequestered by nodes, while the ${ }^{125} \mathrm{I}$ IgM and TcHSA appear to be taken up and retained to a greater extent than the intact ${ }^{125}$ I IgG's.

These differences in uptake kinetics suggest that for delayed imaging of small lymph nodes, TcSbC would

Table 5. ${ }^{125}$ I FT166 (IgM)

\begin{tabular}{|c|c|c|c|c|}
\hline & $20 \mathrm{~min}$ & $1 \mathrm{~h}$ & $2 \mathrm{~h}$ & $4 \mathrm{~h}$ \\
\hline Ipsilateral popliteal node & $102.07 \pm 40.3$ & $64.20 \pm 3.76$ & $62.93 \pm 17.63$ & $32.35 \pm 6.45$ \\
\hline Contralateral popliteal node & $0.128 \pm 0.077$ & $0.051 \pm 0.008$ & $0.243 \pm 0.158$ & $0.056 \pm 0.008$ \\
\hline Ipsilateral femoral node & $0.387 \pm 0.201$ & $2.08 \pm 1.57$ & $0.785 \pm 0.659$ & $0.111 \pm 0.036$ \\
\hline Contralateral femoral node & $0.074 \pm 0.042$ & $0.051 \pm 0.011$ & $0.0676 \pm 0.028$ & $0.044 \pm 0.006$ \\
\hline Liver & $0.037 \pm 0.003$ & $0.061 \pm 0.006$ & $0.050 \pm 0.006$ & $0.045 \pm 0.004$ \\
\hline Blood & $0.087 \pm 0.003$ & $0.129 \pm 0.010$ & $0.134 \pm 0.015$ & $0.144 \pm 0.015$ \\
\hline Ipsilateral leg muscle & $0.008 \pm 0.001$ & $0.015 \pm 0.002$ & $0.015 \pm 0.003$ & $0.013 \pm 0.002$ \\
\hline Contralateral leg muscle & $0.006 \pm 0.0002$ & $0.013 \pm 0.001$ & $0.014 \pm 0.002$ & $0.012 \pm 0.001$ \\
\hline
\end{tabular}

All organ uptakes are expressed as \% kg-injected dose $/ \mathrm{g} \pm 1 \mathrm{SD}$ (following s.c. administration to a hind food pad). 


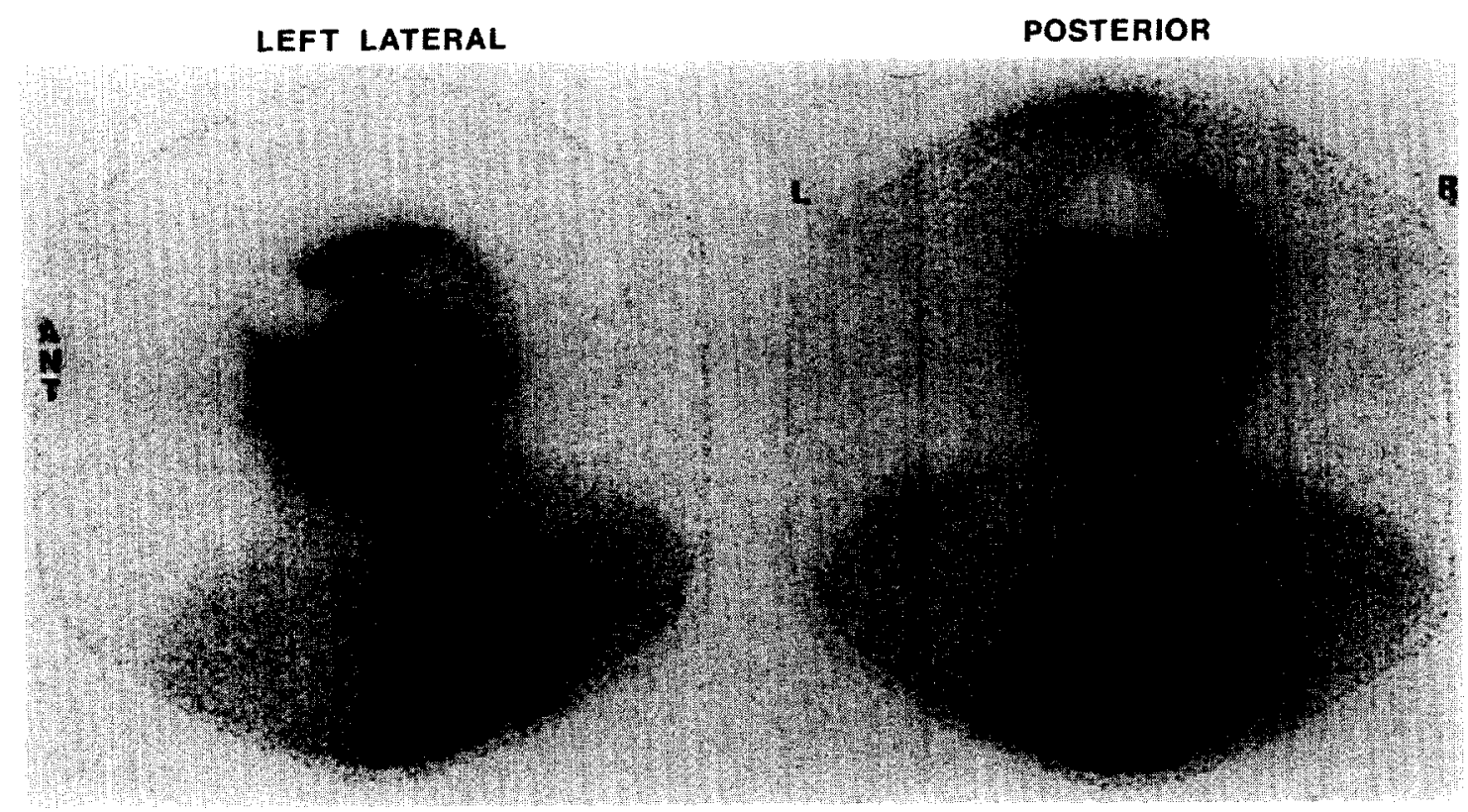

Fig. 2. Left lateral and posterior gamma camera images from approximately 20 min post-injection of $4 \mathrm{mCi}$ of TcHSA about the excisional biopsy site of an anterior scalp melanoma. Note the multiple drainage routes shown on this image. The injection site is shielded with lead. 
be the agent of choice. Despite the higher node/ background ratios for TcSbC, TcHSA in our hands has produced clinically usable lymphoscintigrams of high quality at very early time points (minutes following injection) in patients. While node/blood ratios with TcHSA are lower than with TcSbC, the early node/blood ratio of $>200$ for TcHSA and our excellent clinical images at early times indicate it has considerable utility as a non-specific nodal scanning agent (Eberbach et al., 1987). An example of a clinical scan with TcHSA is shown in Fig. 2. Note that the TcHSA scan shows obvious nodal as well as draining lymphatic channel visualization minutes following the s.c. injection of $4 \mathrm{mCi}$ of TcHSA (Fig. 2). TcSbC scans show mainly the nodes themselves on diagnostic images (Croll et al., 1983; Froelich et al., 1984).

It is of interest that in the rat, the popliteal node uptake of $\mathrm{TcSbC}$ is greater than that of TcHSA at all times. In patients, our clinical impression is that at early time points the uptake of $\mathrm{TcSbC}$ to draining nodes is inferior to that of TcHSA (Eberbach et al., 1987). This apparent discrepancy between the rat data and our clinical observations may be due to the larger distance it is necessary for the scanning agent to traverse from injection site to nodes in man than in rats, or to slower mobilization from the injection site following intradermal or subcutaneous administration.

The relatively high initial and the prolonged retention of the non-specific ${ }^{125}$ I IgM suggests that for purposes of tumor-specific lymphoscintigraphy (i.e. detecting antigen positive tumors in lymph nodes), IgM's may have unacceptably high levels of background activity. Naturally, several other IgM's would need to be evaluated to more definitively address this issue. For the IgG's, however, the background nodal uptake is considerably less, at least at later time points, and the possibility of detecting tumor-specific binding, if present, is correspondingly higher. Despite the lower background with the IgG's, some clinical studies with intact IgG have shown problematically high nodal background levels (Engelstad et al., 1986). Our recent data with ${ }^{131} \mathrm{I}$ antimelanoma antibodies suggests this is less problematic (Wahl et al., 1987).

In conclusion, $\mathrm{TcSbC}, \mathrm{TcHSA}$, and radioiodinated monoclonal antibodies are all taken up into normal lymph nodes after s.c. injection and at early times post-injection this results in high lymph node/background ratios. The uptake of the $\mathrm{TcSbC}$ is highest, the ${ }^{125}$ I IgM next, followed by TcHSA and the ${ }^{125}$ I IgG antibodies. To non-specifically detect lymphatic drainage to lymph nodes, the $\mathrm{TcSbC}$, the iodinated IgM, and TcHSA appear preferable to the iodinated IgGs. The $\mathrm{TcSbC}$ is best suited, based on the animal data, to the detection of small draining lymph nodes due to the high lymph node/background ratio. As agents with lower long-term nodal retention, but definite transport to nodes, the iodinated IgG's appear superior to the other agents. These experi- mental observations should be useful in rationally planning clinical and experimental studies with lymphoscintigraphic agents.

Acknowledgements-Thanks to $\mathrm{Mr}$ Steven Kronberg, $\mathrm{Mr}$ Joe Wissing, Mr Phil Sherman, Ms Linda Laino and Ms Susan Fisher for excellent technical assistance. Dr Barry Wilson's gift of 763.24T antibody is greatly appreciated. Thanks to Mrs Michele Bell for her patience and secretarial assistance. This work was supported in part by PHS grant CA40497 awarded by the Department of Health and Human Services and DOE contract No. DE-AC0276EV002031

\section{References}

Bergqvist, L.; Strand, S.-E.; Persson, B. R. R. Particle sizing and biokinetics of interstitial lymphoscintigraphic agents. Semin. Nucl. Med. 13(1): 9; 1983.

Carrasquillo, J. A.; Bunn, P. A. Jr; Keenan, A. M.; Reynolds, J. C.; Schroff, R. W; Foon, K. A.; Su, M. H.; Gazdar, A. F.; Mulshine, J. L.; Oldham, R. K. et al. Radioimmunodetection of cutaneous T-cell lymphoma with "II In-labeled T101 monoclonal antibody. N. Engl. J. Med. 315(11): 673; 1986.

Croll, M. N.; Brady, L. W.; Dadparvar, S. Implications of lymphoscintigraphy in onoclongic practice: Principles and differences vis-à-vis other imaging modalities. Semin. Nucl. Med. 13(1): 4; 1983.

DeLand, F. H.; Kim, E.; Goldenberg, D. M. Lymphoscintigraphy with radionuclide labeled antibodies to carcinoembryonic antigen. Cancer Res. 40: 2997; 1980.

Eberbach, M. A.; Wahl, R. L.; Argenta, L. C.; Froelich, J.; Niederhuber, J. E. Utility of lymphoscintigraphy in directing surgical therapy of head, neck and upper thorax melanomas. Surgery 102(3): 433-442; 1987.

Engelstad, B. L.; Spitler, L. E.; Del Rio, M. J.; Ramos, E. C.; Rosendorf, L. L.; Reinhold, C. E.; Khentigan, A.; Huberty, J. P.; Corpuz, W.; Lee, H. M. et al. Phase 1 immunolymphoscintigraphy with an In-111 labeled antimelanoma monoclonal antibody. Radiology 161: 419; 1986.

Froelich, J. W.; Swanson, D. P.; Mast, C.; Wahl, R. L.; Lahti, D. S. Tc-99m HSA-The ideal agent for cutaneous lymphoscintigraphy. Radiology 153(P): 316; 1984.

Hopf, U.; Zum Buschenfelde, K. H. M.; Dierich, M. Demonstration of binding sites for IgG Fc and the third complement component (C3) on isolated hepatocytes. J. Immunol. 117: 639; 1976.

Laemmli, V. K. Cleavage of structural proteins during assembly of the head of bacteriophage T4. Nature 222 : $680 ; 1970$.

Liebert $M$. Unpublished data.

Wahl, R. L.; Geatti, O.; Liebert, M.; Beers, B.; Jackson, G.; Laino, L.; Kronberg, S.; Wilson, B. S.; Beierwaltes, W. H. Kinetics of intralymphatically delivered monoclonal antibodies. J. Nucl. Med. 26(5): P55; 1985.

Wahl, R. L.; Liebert, M.; Biesman, B.; Roberts, J.; Jackson, G.; Kronberg, S.; Laino, L. Production and characterization of a murine monoclonal antibody reactive with ovarian and other epithelial carcinomas. Proc. $A A C R$ 27: $355 ; 1986$.

Wahl, R. L.; Liebert, M.; East, J.; Headington, J.; Coon, W.; Shulkin, B. L.; Niederhuber, J.; Wilson, B.; Natale, R.; Davenport, R.; Swanson, N. Lymphoscintigraphy in melanoma: Experience with a monoclonal antibody cocktail. J. Nucl. Med. 28(4): 713; 1987.

Wahl, R. L.; Sherman, P.; Fisher, S. The effect of specimen processing on radiolabeled monoconal antibody biodistribution. Eur. J. Nucl. Med. 9(8): 382; 1984.

Wallis, J. W.; Fisher, S.; Wahl, R. ${ }^{99 m}$ Tc uptake by lymph nodes following infiltration: Clinical and laboratory evaluation. Nucl. Med. Commun. 8: 357; 1987. 
Weinstein, J. N.; Steller, M. A.; Keenan, A. M.; Covell, D. G.; Key, M. E.; Sieber, S. M.; Oldham, R. K.; Hwang, K. M.; Parker, R. J. Monoclonal antibodies in the lymphatics: Selective delivery to lymph node metastases of a solid tumor. Science 222: 423; 1983.

Wilson, B. S.; Giacomini, P.; Imai, K.; Natali, P. G.;
Nakanishi, T.; Ruberto, G.; Ferrone, S. Human melanoma associated antigens identified with monoclonal antibodies: Structural profile and potential usefulness in immunodiagnosis and immunotherapy. La Ricerca in Clinica e in Laboratorio 12: 517; 1982. 